\title{
Optimal sample length for calculating transfer functions from discrete experimental data
}

Scott Taylor* Nicholas Haritos* Krish Thiagarajan ${ }^{\dagger}$

(Received 27 October 2004, revised 2 May 2005)

\begin{abstract}
Transfer functions are useful tools in observing the behaviour of non-linear systems. Transfer functions convert an input signal into an output signal, and for non-linear systems can be calculated separately for each order of response using the Volterra series. The Volterra series quantifies the linear and non-linear responses separately for systems with either Gaussian or non-Gaussian inputs, and is particularly useful when calculating transfer functions from experimental data, as the calculations can be performed using a discrete frequency domain format. The application of the Volterra series to discrete experimental data requires careful consideration of various factors that impact
\end{abstract}

${ }^{*}$ Department of Civil and Environmental Engineering, The University of Melbourne, Melbourne, AustraliA. mailto:Scott_Taylor@ghd.com.au and mailto:nharitos@unimelb.edu.au

${ }^{\dagger}$ School of Oil and Gas Engineering, The University of Western Australia, Perth, Australia. mailto:krish.thiagarajan@uwa.edu.au

See http://anziamj.austms.org.au/V46/CTAC2004/Tayl for this article, (C) Austral. Mathematical Soc. 2005. Published June 29, 2005. ISSN 1446-8735 
on the successful calculation of transfer functions. The single largest problem faced when using discrete experimental data is the difficultly presented when determining the optimal sample length of the data adopted during the calculation process. Equal lengths of sample data are extracted from each individual record to calculate averaged input and output spectra. When using experimental data of finite record length, a trade off between the number of sample lengths obtained from each record and the frequency interval of the resulting transfer functions occurs. Here we explore those factors that lead to the selection of an optimal sample length. We begin with an overview of the Volterra series approach, and how experimental data can be used to calculate transfer functions. The factors that influence the selection of the optimal sample length are described and methods outlined that ensure the most meaningful results are obtained. We demonstrate these methods using the results from an experimental procedure performed on a model Tension Leg Platform.

\section{Contents}

1 Introduction

2 The Volterra series

3 The optimal sample length

4 Principal component analysis

C578

5 Results

C579

6 Conclusions

C583

References 


\section{Introduction}

Transfer functions are particularly useful in observing non-linear behaviour, as they convert an input signal into output signals at each order of response. In the time domain, an input signal is convolved with an impulse response transfer function to give the output signal. In the frequency domain an input spectrum is simply multiplied by a frequency response transfer function to give the output spectrum [3]. Usually, convolution in the time domain is computationally intensive making the use of transfer functions in the frequency domain is more attractive.

The Volterra series usefully solves for transfer functions from discrete experimental data. This approach allows the quantification of the linear and non-linear (for example, quadratic, cubic, etc.) components separately. Section 2 overviews the Volterra series, including its origin and development to its most general form. When it is extended to discrete experimental data, selection of the optimal sample length for treatment in the Volterra series becomes critical. Two opposing factors that influence the size of the sample length are discussed in Section 3: the number of sample lengths that can be obtained from a finite record; and the size of the frequency interval of the resulting system. One technique that is successful in ensuring the minimal number of expected values required to form an accurate solution is principal component analysis. Section 4 outlines this technique and its application to the Volterra series. Finally, in Section 5, results from an experimental procedure on a model Tension Leg Platform (TLP) demonstrate the outlined methods.

\section{The Volterra series}

Tick [5] was the first to calculate transfer functions using a modified version of Volterra's series that used input $(X(f))$ and output $(Y(f))$ spectra, as 
opposed to time series inputs first proposed by Volterra. Tick calculated transfer functions from cross-power spectra and cross-bispectra, although the work was limited to Gaussian excitations and the output was calculated up to the second-order response only. The Volterra series up to the $N$ th order, in a continuous frequency domain form [4], is

$$
\begin{aligned}
& Y(f)=\int_{-\infty}^{\infty} H_{1}\left(f_{1}\right) X\left(f_{1}\right) \delta\left(f-f_{1}\right) d f_{1} \\
& +\int_{-\infty}^{\infty} \int_{-\infty}^{\infty} H_{2}\left(f_{1}, f_{2}\right) X\left(f_{1}\right) X\left(f_{2}\right) \delta\left(f-f_{1}-f_{2}\right) d f_{1} d f_{2} \\
& +\cdots \\
& +\int_{-\infty}^{\infty} \cdots \int_{-\infty}^{\infty} H_{N}\left(f_{1}, \ldots, f_{N}\right) X\left(f_{1}\right) \ldots X\left(f_{N}\right) \delta\left(f-f_{1} \ldots-f_{N}\right) d f_{1} \ldots d f_{N}
\end{aligned}
$$

This equation calculates the total output spectrum $(Y(f))$ from the input spectrum $(X(f))$ multiplied by transfer functions at each order of response, where

- $H_{1}\left(f_{1}\right)$ is the first-order frequency response transfer function,

- $H_{2}\left(f_{1}, f_{2}\right)$ is the second-order frequency response transfer function,

- $H_{N}\left(f_{1}, \ldots, f_{N}\right)$ is the $N$ th order frequency response transfer function, and

- $\delta(\cdot)$ is the Dirac delta function.

Using vector notation, define a row vector $\boldsymbol{H}_{2}(f)$ as a collection of all the second-order transfer functions at discrete frequency pairs, $H_{2}\left(f_{1}, f_{2}\right)$ for example, where $f_{1}+f_{2}=f$. The row vector $\boldsymbol{H}_{N}(f)$ is a collection of all the $N$ th order transfer functions at discrete frequencies, $H_{N}\left(f_{1}, f_{2}, \ldots, f_{N}\right)$ for example, where $f_{1}+f_{2}+\cdots+f_{N}=f$. Similarly, define the input 
spectra in terms of column vectors, where $\boldsymbol{X}_{2}(f)$ is a collection of all the pairs of inputs $X\left(f_{1}\right) X\left(f_{2}\right)$, where $f_{1}+f_{2}=f$. And finally, the column vector $\boldsymbol{X}_{N}(f)$ is a collection of all the inputs $X\left(f_{1}\right) X\left(f_{2}\right) \cdots X\left(f_{N}\right)$, where $f_{1}+f_{2}+\cdots+f_{N}=f$. Using these vectors express Equation (1) in matrix form as

$$
Y(f)=\boldsymbol{H}(f) \boldsymbol{X}(f)+\varepsilon(f),
$$

where

$$
\begin{aligned}
& \boldsymbol{H}(f)=\left[\begin{array}{llll}
H_{1}(f) & \boldsymbol{H}_{2}(f) & \cdots & \boldsymbol{H}_{N}(f)
\end{array}\right], \\
& \boldsymbol{X}(f)=\left[\begin{array}{c}
X_{1}(f) \\
\boldsymbol{X}_{2}(f) \\
\vdots \\
\boldsymbol{X}_{N}(f)
\end{array}\right] \text {. }
\end{aligned}
$$

When solving Equation (2) determine the optimum transfer functions by minimising the modelling error $\varepsilon(f)$ between the observed output $Y(f)$ and the model output (calculated by $\boldsymbol{H}(f) \boldsymbol{X}(f)$ ). In particular, since the Volterra model is linear with respect to the transfer functions, apply the method of least squares. This requires Equation (2) to be multiplied by the complex conjugate of the input vector $\boldsymbol{X}^{*}(f)$ and the expected values of each side of the equation to be found. The function $E[\cdot]$ describes an expected value or statistical averaging process, where an estimate of the density function is determined by obtaining an average of a data record from numerous short segments. The superscript $T$ denotes the transpose of the vector to give

$$
E\left[\boldsymbol{X}^{*}(f) Y(f)\right]=E\left[\boldsymbol{X}^{*}(f) \boldsymbol{X}^{T}(f)\right] \boldsymbol{H}^{T}(f) .
$$

When $E\left[\boldsymbol{X}^{*}(f) \boldsymbol{X}^{T}(f)\right]^{-1}$ exists, rearrange Equation (3) into the form

$$
\boldsymbol{H}^{T}(f)=E\left[\boldsymbol{X}^{*}(f) \boldsymbol{X}^{T}(f)\right]^{-1} E\left[\boldsymbol{X}^{*}(f) Y(f)\right] .
$$




\section{The optimal sample length}

Typically, for discrete data (whether computer generated or obtained from experiments), inaccuracies occur when the Fourier transform maps the data from the time to the frequency domain. However, the nature of discrete data means that unlike a continuous system, a finite record of discrete data is collected at specific values a constant width apart. Due to these characteristics, spectral leakage occurs when the response lies between the frequency intervals of the transformed data.

Whereas the general trend of the real signal can be observed, by digitising the system, the exact solution is lost. One process that can reduce spectral leakage is windowing, where the use of tapered windows reduces the influence of the tails of the discrete data [2]. However, windows are largely developed for linear systems, and while research continues into a form applicable to non-linear systems, herein no windows are used on the data. So, the smaller the frequency interval the lower the influence of spectral leakage, and a more accurate observation of the characteristics of the system can be made.

For experimental data, noise causes additional inaccuracies. Therefore, a way to alleviate this problem is to find the expected value of the input and output spectra. When calculating the transfer functions using Equation (4) an expected value is taken to form the matrices $E\left[\boldsymbol{X}^{*}(f) \boldsymbol{X}^{T}(f)\right]$ and $E\left[\boldsymbol{X}^{*}(f) Y(f)\right]$. This ensures the final representation of these matrices is an averaged or expected value of all the data lengths available. The more sample lengths used to form the expected value, the greater the confidence that can be placed upon the response.

However, herein lies the problem of determining the length of data taken from a fixed length record required to form the sample lengths. When dealing with discrete experimental data, the total record lengths are always finite. Therefore, the choice of the optimal sample length is based around a trade off between small enough frequency intervals versus obtaining sufficient sample 
lengths from a record of finite length for adequate spectral representation.

\section{Principal component analysis}

One technique that can result in significant savings in the number of expected values required to obtain an accurate result is the use of principal component analysis to invert the input matrix $E\left[\boldsymbol{X}^{*}(f) \boldsymbol{X}^{T}(f)\right]$ used in Equation (4). The near singularity of the matrix means that directly inverting it rarely leads to actual solutions. An alternative approach suggested by Birkelund et al. [1] is that principal component analysis be used to solve for the inverse of the input matrix $E\left[\boldsymbol{X}^{*}(f) \boldsymbol{X}^{T}(f)\right]$. Since the matrix is Hermitian, decompose it into the form

$$
E\left[\boldsymbol{X}^{*}(f) \boldsymbol{X}^{T}(f)\right]=\boldsymbol{U} \boldsymbol{D} \boldsymbol{U}^{H},
$$

where the superscript $H$ represents the Hermitian of the matrix. Denoting the eigenvalues by $\lambda_{i}$ and the corresponding eigenvectors by $\boldsymbol{u}_{i}$, matrix $\boldsymbol{D}=$ $\operatorname{diag}\left\{\lambda_{1}, \lambda_{2}, \ldots, \lambda_{n}\right\}$ and the unitary matrix $\boldsymbol{U}=\left[\boldsymbol{u}_{1}, \boldsymbol{u}_{2}, \ldots, \boldsymbol{u}_{n}\right]$.

The form of the matrix is such that many of the columns are very nearly linearly dependent, identified by the small magnitude of the columns' eigenvalues. Subsequently, if those eigenvalues (and corresponding eigenvectors) that are deemed to be very nearly linearly dependent are removed from the matrix, then only those components that have a significant impact are retained. The remaining eigenvalues and eigenvectors (represented by $\boldsymbol{D}_{r}$ and $\boldsymbol{U}_{r}$ respectively, where the subscript $r$ represents the number of columns retained) are used to approximate the matrix as

$$
E\left[\boldsymbol{X}^{*}(f) \boldsymbol{X}^{T}(f)\right] \approx \boldsymbol{U}_{r} \boldsymbol{D}_{r} \boldsymbol{U}_{r}^{H},
$$

so that

$$
E\left[\boldsymbol{X}^{*}(f) \boldsymbol{X}^{T}(f)\right]^{-1} \approx \boldsymbol{U}_{r} \boldsymbol{D}_{r}^{-1} \boldsymbol{U}_{r}^{H}
$$


There is a direct link between the magnitude of an eigenvalue and the relative percentage of the variance that the eigenvalue and corresponding eigenvector account for in the matrix. So by utilising only the significant eigenvalues and eigenvectors to estimate the matrix, all the nearly linear dependent components will be removed. Birkelund et al. [1] obtained significantly better results when using principal component analysis to invert the input matrix in the Volterra series. In particular, they found that the method reduces the number of sample lengths required to achieve an adequate convergence in the relative squared error term. This is due to the fact that when the matrix is inverted, the use of principal component analysis eliminates effects of near singularity of the matrix, and subsequently gives a fair representation of its inverted form.

\section{$5 \quad$ Results}

To investigate the Volterra series using principal component analysis, and the impact of the sample length on the transfer functions, data was collected from an experimental procedure performed on a Tension Leg Platform (TLP). TLPS are a type of offshore structure that contains a buoyant platform (consisting of pontoons and columns) connected to the seabed by four tendons located at each corner of the platform. This design is more flexible than traditional jacket based offshore structures, and as a consequence it has been found that due to second-order effects a non-linear sum-frequency response occurs at the heave natural frequency. As part of an investigation into identifying this phenomenon, a model TLP was subject to regular and irregular waves in the testing facilities at the Institute for Marine Dynamics in Canada. Typically, each test contained 25,000 data points taken at a time interval of 0.02 seconds. The input data was taken as the wave height profile, and the output data as the vertical (heave) displacement. To verify the test results, a specialised computer program, WAMIT, was used to model the TLP under wave loading. WAMIT is a second-order radiation/diffraction program 
TABLE 1: The correlation coefficients between the experimental results of different sample lengths and the WAMIT results for the linear transfer functions.

\begin{tabular}{|c|c|}
\hline Sample length & Correlation coefficient \\
\hline 256 & 0.056 \\
512 & 0.092 \\
1024 & 0.591 \\
2048 & 0.450 \\
\hline
\end{tabular}

developed for the analysis of the interaction of surface waves with offshore structures. It has been successful in offshore research to predict the response of model and prototype structures.

When dealing with dynamic systems that typically display a peak value at the natural frequency, the frequency resolution must be adequate to ensure the characteristics are sufficiently captured by the transfer functions. To demonstrate the impact of using different sample lengths, the linear transfer functions for the model are shown in Figure 1 while the correlation coefficients between the experimental and WAMIT results are listed in Table 1. These transfer functions are based on an average of the entire regular and irregular wave tests. See in Table 1 that for the linear transfer functions a sample length of 1024 provides the best representation of the system's characteristics when compared to the WAMIT results. That is, the frequency resolution for sample lengths of size 256 and 512 are too coarse and the peak value at the natural frequency is not captured. Longer sample lengths (of size 2048 and possibly greater) would likely not provide a sufficient number of individual samples when finding the expected values necessary to reduce the effects of noise, for the record lengths collected in this investigation.

Principal component analysis assists with the calculation of the transfer functions. Note: in Figure 1 when the size of the sample length was investigated, principal component analysis was used, in which all eigenvalues higher than 0.005 times the maximum eigenvalue were retained. If the ma- 


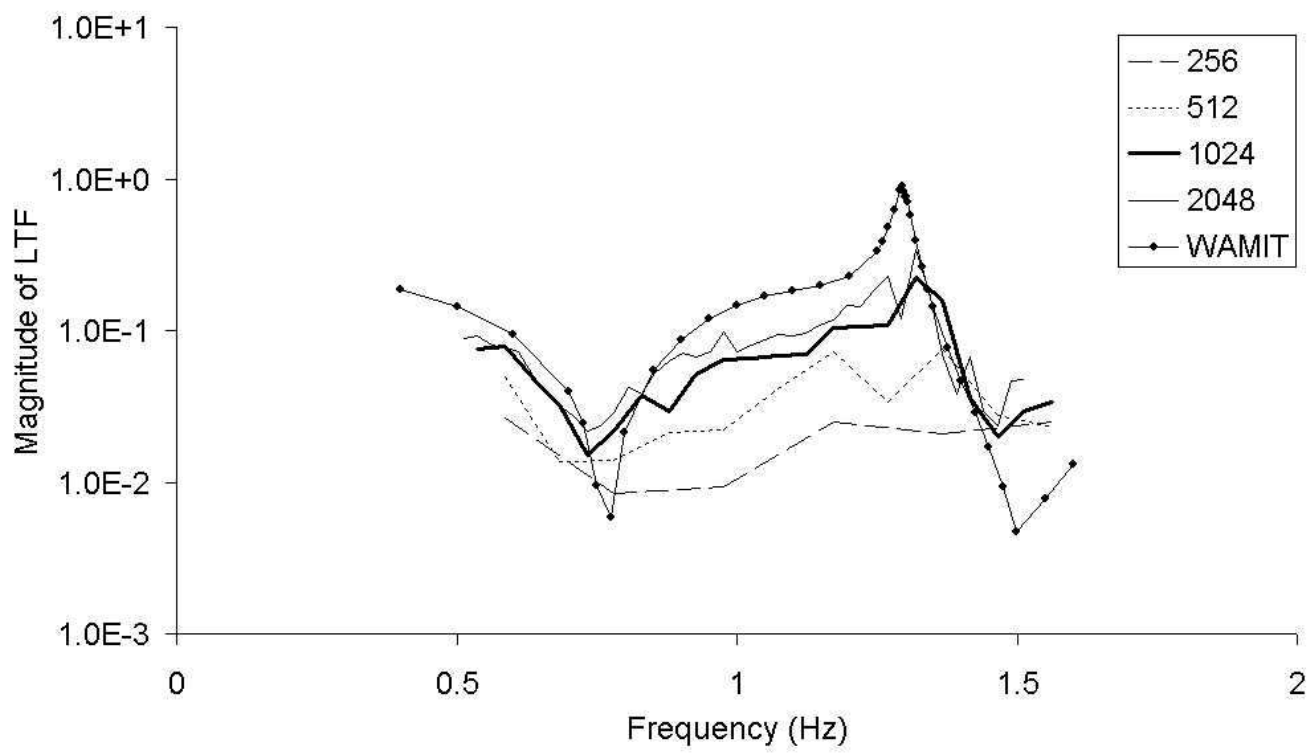

FiguRE 1: Linear transfer function calculated with varying sample lengths.

TABLE 2: The correlation coefficients between the experimental results with a different number of principal components retained and the WAMIT results for the linear transfer functions.

\begin{tabular}{|c|c|}
\hline Size of principal components retained & Correlation coefficient \\
\hline 0.01 & 0.481 \\
0.005 & 0.591 \\
0.001 & 0.280 \\
0.0005 & 0.338 \\
\hline
\end{tabular}




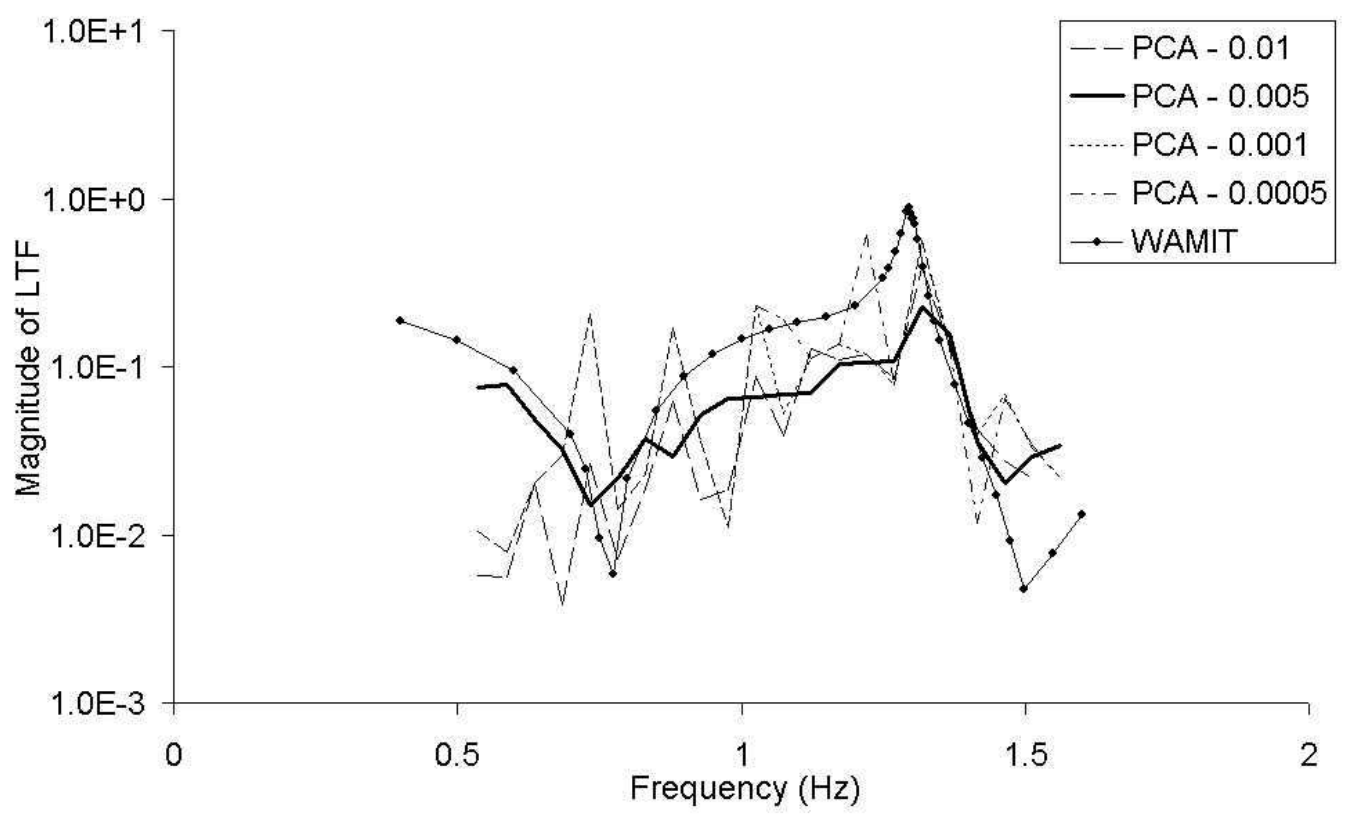

FIGURE 2: Linear transfer function calculated with a varying number of eigenvalues retained. 
trix $E\left[\boldsymbol{X}^{*}(f) \boldsymbol{X}^{T}(f)\right]$ was inverted directly, the near singularity of the matrix would mean the results would be meaningless (and were found to be around $10^{10}$ times larger in magnitude). However, the number of eigenvalues to be retained can also influence the form of the transfer functions. The number of eigenvalues to be retained was determined by comparing individual eigenvalues to the maximum eigenvalue. From Figure 2 and Table 2, it is possible to see that the best results arise from when those eigenvalues that are larger than 0.005 times the maximum are retained.

Therefore, using the generalised Volterra series, with principal component analysis to invert the input matrix with eigenvalues greater than 0.005 times the maximum retained, and a length of 1024 data points used to form individual samples, the resulting linear and quadratic transfer functions are shown in Figures 3 and 4 respectively. In these graphs the irregular wave tests are depicted by lines as the results are obtained from a range of frequencies for these tests, whereas the regular wave tests are depicted as points as their results are obtained at single frequencies. Comparing the test results to the WAMIT transfer functions, a reasonable match is achieved, showing that a sufficiently optimal sample length was arrived at using this approach.

\section{Conclusions}

Transfer functions allow the characteristics of dynamic non-linear systems to be easily observed. Using the Volterra series, transfer functions at each order of response can be calculated from input and output data. However, the successful application of the Volterra series to discrete experimental data requires careful consideration of the optimal sample length to be used to form the expected values. Determination of the optimal sample length requires a trade off between a small enough frequency resolution, and the number of sample lengths that can be obtained from a finite record length. If the frequency interval is too large, spectral leakage will occur and the resolution 


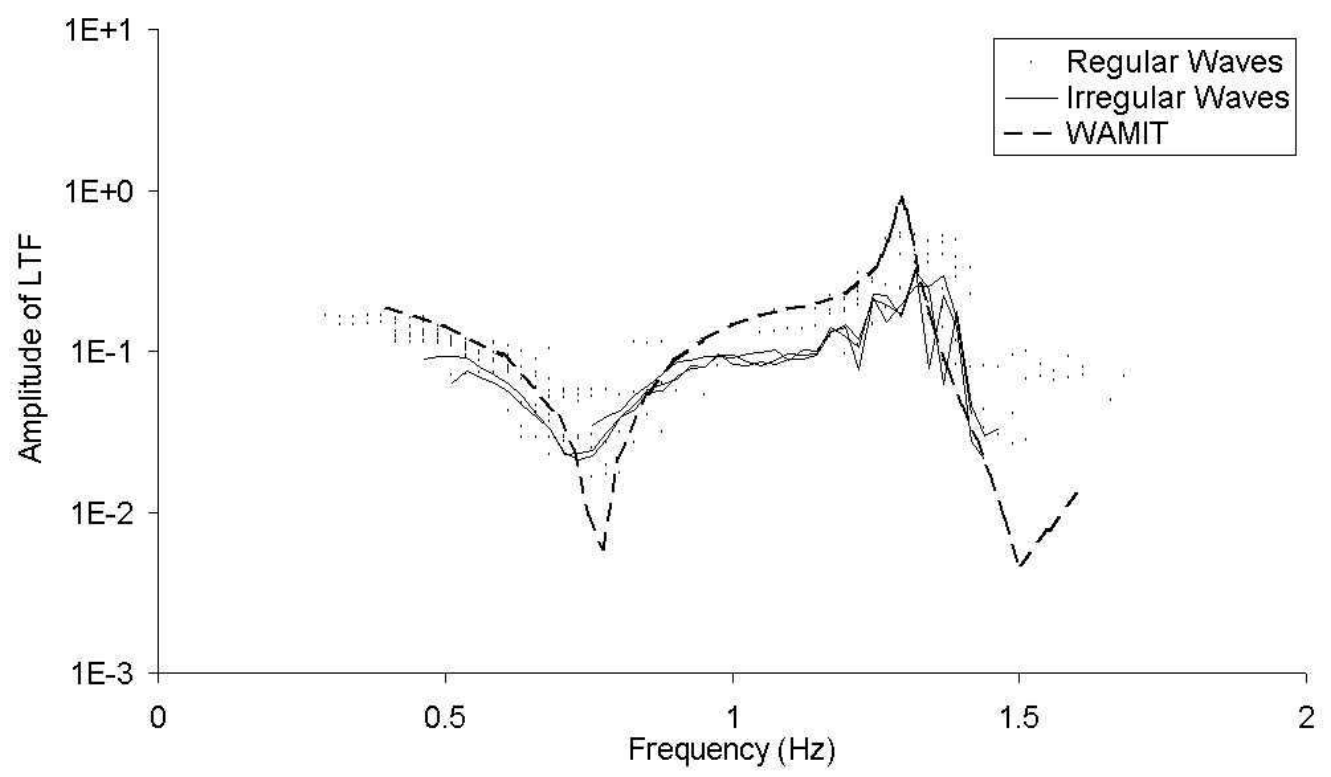

FigURE 3: Linear transfer function. 


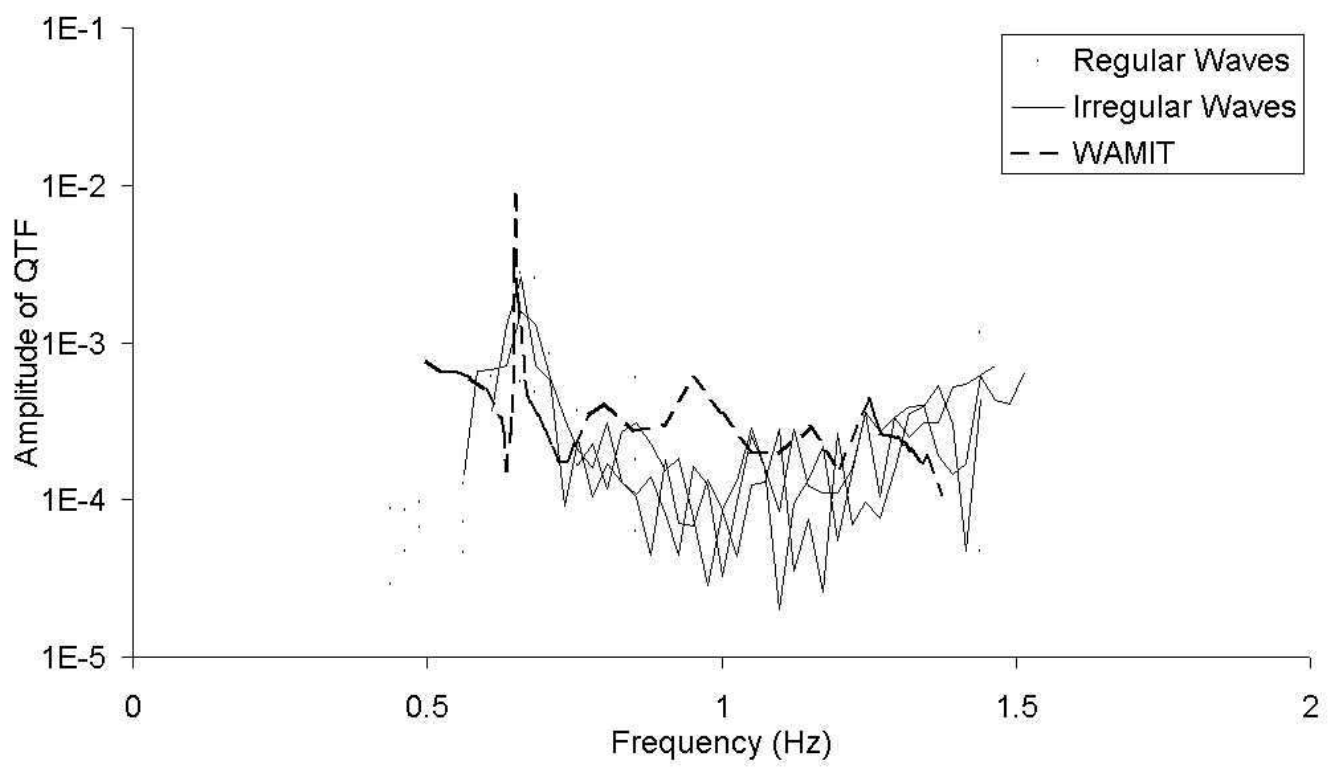

FIGURE 4: Quadratic transfer function. 
of the transfer functions will be too course. If an insufficient number of sample lengths are used to form the expected values, errors introduced through noise may be problematic.

A technique that reduces the number of sample lengths necessary to obtain an accurate representation of the transfer functions is principal component analysis. When calculating the transfer functions the input matrix must be inverted which causes problems, as it is very nearly singular. Therefore, principal component analysis allows the matrix to be represented by those eigenvalues and eigenvectors that constitute the major components of the matrix, by eliminating the linear dependent eigenvalues.

Using these techniques transfer functions were calculated from experimental data collected from a model TLP. Principal component analysis was utilised by retaining all eigenvalues that were greater than 0.005 times the size of the maximum eigenvalue. Sample lengths of 1024 data points were used to form the expected values from the records. A good match was achieved between the experimental and computational results demonstrating that a sufficiently optimal sample length of data was adopted.

Acknowledgment: We acknowledge and thank Dr. Bruce Colbourne and Dr. Emil Baddour from The Institute for Marine Dynamics at The National Research Council of Canada, and Dr. Jinshu Xia from The Australian Maritime College for providing access to the testing equipment and assisting with the testing procedure, respectively.

\section{References}

[1] Y. Birkelund, A. Hanssen and E. J. Powers. On the Estimation of Non-Linear Volterra Models in Offshore Engineering. International 
Journal of Offshore and Polar Engineering, 13:12-20, 2003. C578, C579

[2] F. J. Harris. On the Use of Windows for Harmonic Analysis with the Discrete Fourier Transform. Proceedings of the IEEE, 66:51-83, 1978. C577

[3] K. I. Kim and E. J. Powers. A Digital Method of Modelling Quadratically Non-Linear Systems with a General Random Input. IEEE Transactions on Acoustics, Speech and Signal Processing, 36:1758-1769, 1988. C574

[4] S. R. Taylor, N. Haritos, and K. P. Thiagarajan. Modelling the Heave Response of Tension Leg Platforms Using the Volterra Series. 22nd International Conference on Offshore Mechanics and Arctic Engineering, 1:71-79, 2003. C575

[5] L. J. Tick. The Estimation of Transfer Functions of Quadratic Systems. Technometrics, 42(7):1746-1765, 1961. C574 